\title{
Capability studies, helpful tools in process quality improvement
}

\author{
Carmen Simion ${ }^{1, *}$ \\ ${ }^{1}$ Lucian Blaga University of Sibiu, Department of Industrial Engineering and Management, 550025 \\ Emil Cioran street 4, Sibiu, Romania
}

\begin{abstract}
Ability of processes to meet customer'quality requirements has become essential for providing competitive advantages such as cost savings, reducing the number of nonconfoming products or increasing customer satisfaction. This paper aims to conduct a capability study for a swaging process (that ensures an assembly dimension) of a key product characteristic (the outer diameter of a new part), related to the concept of capability and performance indices and how these metrics can be used and interpreted to become powerful tools for decision making. To achieve the goal of the paper, the following key aspects were analyzed: capability of the measurement system capability (gage $R \& R$ ), production equipment (machine) capability and process capability/performance during the first serial production. The analysis was performed with Minitab ${ }^{\circledR}$ 17, the most commonly used software for quality improvement.
\end{abstract}

\section{Process variation}

As a matter of fact, in any process, regardless of how well designed or carefully maintained it is, a certain amount of inherent or natural variability will always exist [1]. This type of variation is the cumulative effect of many small causes, named common, chance or random causes. These types of causes produce a consistent and predictable type of variation over time. A process that is operating with only common causes of variation present is said to be a stable process or in a state of statistical control. Sometimes, dominant sources of variation may appear, that are not part of the process and they are named as special or assignable causes. These types of causes are not predictable and are sporadic in nature.

It is important to know what type of variation is present in a process, because the action one takes for the process improvement depends on the type of variation. If special causes of variation are present, they should be first identified and then eliminated; the next step is to reduce common cause of variation.

Competitive companies understand that satisfying the needs of customers and running a successful operation requires processes that are predictable and consistent. The variation which is present in a process (whether it is a business or a manufacturing process) is the main cause of the quality problems, so measuring variability and finding ways to reduce it, is essential for the success of any company.

* Corresponding author: carmen.simion@ulbsibiu.ro 
In a manufacturing process, the observed process variation comes from two sources [2]: variation due to the differences between parts made by the process (part-to-part variation) called actual process variation and variation due to the measurement variation (measurement system or gage/gauge variation) i.e. imperfections in taking measurements.

There are numerous methods to control and reduce manufacturing process variation, e.g. statistical process control (SPC) tools, measurement system analysis (MSA) tools, six sigma methods, Taguchi's design of experiments [3].

\section{Capability studies}

An important initial step in any process quality improvement is to quantify the capability of the product realization process (manufacturing process). Capability is defined [4] as the ability of the process to realize a characteristic that will fullfil the requirements for that characteristic. According to ISO standards, any organization shall measure the characteristics of the product to verify that product requirements have been met carried out at appropriate stages of the product realization process.

The capability study is an important concept to understand [5], which has gained wide acceptance around the world as a tool for process quality improvement. A process capability study is a set of calculations used to evaluate wheather a process (generally, a sistem) is statistical able to meet a set of specifications or requirements.

Automotive Industry Action Group [6] distinguishes between long-term and short-term capability studies; a short-term capability study (e.g., machine capability study) evaluates the parts manufactured in one continuous production run; a long-term capability study (e.g., manufacturing process capability study) evaluates the parts manufactured over a longer time which is representative of the variation encountered in series production.

Capability studies must be done not only to the machine on which the manufacturing process is running and to the manufacturing process, but also to the measurement system used in taking measurements of product characteristics.

Capability studies are performed for a new or changed production process (including assembly) in order to verify initially a preliminary capability and then over time to obtain additional inputs for controlling the process [7].

A capability study is summarized in indices; the purpose of these measures is to show the system's ability to meet its requirements, respectively to determine whether the system is capable of producing products which are within the customer specifications [8].

There are many capability indices that have been developed through time and they are divided into: capability indices of the machine, capability indices of the measurement system and capability/performance indices of the manufacturing process. The basic commonly used indices are presented in the following.

\subsection{Machine capability study}

Machine capability study is a short-term study and refers exclusively to the ability of a machine to produce parts. A machine capability study includes the following steps [9]:

- Manufacturing of a representative number of parts (minimum 50, if possible 100) in a continuous, uninterrupted production run;

- Measurement of the part characteristic(s);

- Calculation of capability indices.

Machine capability is summarized in $\mathrm{Cm}$ and $\mathrm{Cmk}$ indices: $\mathrm{Cm}$ represents the possible potential of the machine when no systematic factors exist; Cmk describes the practical state of the machine with reference to how centered mean value offsets are considering upper and lower specification limits. A machine is capable if $\mathrm{Cmk} \geq 1.67$. 


\subsection{Measurement system capability study}

When one bases decisions on production data, the accuracy of that data is critical [2]. If the measurement system has too much variation, it will adversely influence the decisions. The measuring equipment (gage, gauge), the appraiser (evaluator, inspector) and the measuring procedure determine the variation due to the measurement system.

Information on how to conduct a measurement system analysis (measurement system capability study) are given in $[2,10]$. An usual procedure is to do a repeatability and reproducibility (gage $R \& R$ ) study. Repeatability is the variation in the measurements obtained by one appraiser while measuring the same item repeatedly; this is also called equipment variation. Reproducibility is the variation in the measurements obtained by several appraisers while measuring the same item repeatedly; this is called appraiser variation, too. Repeatability and reproducibility represent measurement system variability.

An ideal measurement system should not have any variation, but this is impossible; a common rule of thumb is that gage $R \& R$ variation should be less than $10 \%$ of the smaller of either the process variation or tolerance (specification).

\subsection{Manufacturing process capability study}

The purpose of process capability study is to obtain a better understanding of process variation and to quantify how well the process can produce parts fulfilling the requirement.

Manufacturing process capability study can be done in short-term as well as in long-term by using control charts. In a short term study, the interest is in evaluating inherent process variation; many factors such as tools, operators, environment etc. do not change appreciably during study. Long term process capability study allows effects of many factors in the process such as operators, raw material, tools etc [11].

When one measures relatively less number of parts (about 50 parts are recommended) short-term capability is evaluated; for a long-term capability study, a minimum of 100-125 parts must be measured. A process capability study includes the following steps $[9,12]$ :

- Select and examined parts from series production in "rational" samples; for short-term study at least 10 subgroups should be evaluated; for long-term study at least 20-25 subgroups should be evaluated. The preferred subgroup size is $n=5$ parts;

- Measure part characteristics and record the results along with production sequence;

- Statistical evaluation of the data: statistical distribution and stability;

- Calculate process capability and performance indices.

Most widely used indices are process capability indices $\mathrm{Cp}, \mathrm{Cpk}$ and process performance indices Pp, Ppk [5]. The difference between these two indeces category is the way the process standard deviation is calculated. Process capability indices measures how capable the process is to satisfy specification limits by using the "within subgroup variation" which analyzes the shifts and drifts within subgroup. Higher $\mathrm{Cp}$ and $\mathrm{Cpk}$ value, more capable the process is. Many companies use $\mathrm{Cpk} \geq 1.33$ as benchmark value to determine whether the process is capable. Process performance indices Pp and Ppk consider "between (overall) subgroup variation" and they take into account the shifts and drifts between subgroups. Ppk $\geq 1.67$ is a common value for most companies.

There are two critical assumptions to consider when performing process capability analyses. If they are not met, the resulting statistics may be highly unreliable [13]:

- The output data must follow a normal distribution. There are several analysis methods used to determine if the data are normally distributed or not: graphical methods as histogram and normal probability plot and analytical methods, goodness-of-fit tests.

- The process must be in statistical control (stable). Control charts are used to verify if a process is under statistical control. 


\section{Case study}

Before a serial production of a certain product type, the manufacturing process must first be omologated in order to better meet customer requirements and this calls for a preliminary capability study. The aim is to see that the manufacturing process is capable of production within specifications, which implies a certain minimum value for capability indices.

Preliminary capability study includes machine capability study, measurement system capability analysis and preliminary process capability study. After the process has been omologated (preliminary capability was demonstrated) production can be conducted. During production, the process will be regularly reviewed by using capability studies, to ensure an economic production level and of adequate quality.

To determine the general ability of the manufacturing process to meet customer specifications, capability indices of machine and gage and preliminary performance indices of process for all critical product characteristics should be calculated. Then from time to time, over the life of the product, process capability indices must be generated [14].

\subsection{Company and analyzed product}

In last years, Sibiu has attracted significant investment in automotive industry and some of the largest manufacturers of automotive parts in the world have opened production facilities in the area. One of these companies is CMP, expert in automotive anti-vibration systems.

CMP designs, develops and manufactures anti-vibration components, products which are part of the chassis systems for passengers and commercial vehicles. They can be categorised by functionality as products which are found in suspension systems (these are joints elements for front and rear axle mechanisms) and products which elastically support units in the structure of the vehicle, emphasising among them the engine mounts.

The case study presented in the paper analyzes the swaging process (a process that ensures an assembly dimension) for a new product "rubber bushing with mold bonded metal sleeves" (part number 14001). The critical quality characteristic of the product is the outer diameter, after the swaging process (Fig. 1).
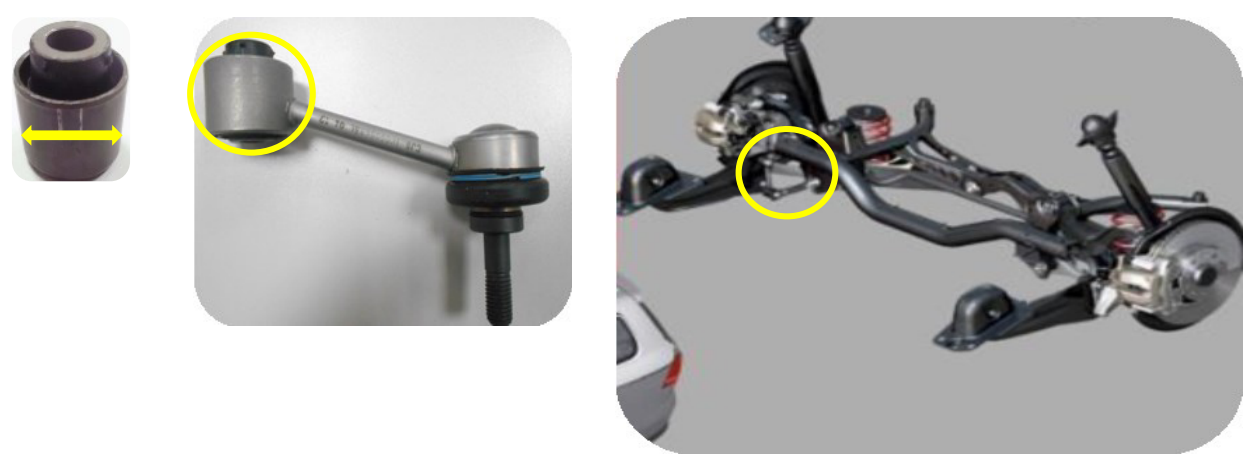

Fig. 1. Rubber bushing with mold bonded metal sleeves

The part is calibrated from initial diameter of $\varnothing 27.3+/-0.1$ to a final diameter of $\varnothing 26$ $+0.45 /+0.25$ on an automatic swaging machine with sectors, whose primary function is to reduce the outer diameter of the part with approximately $1 \mathrm{~mm}$ and also to compress the vulcanized rubber between the metal (inner and outer insert) sleeves of the bushing.

To achieve measurements, a digital caliper with a resolution of $0.01 \mathrm{~mm}$ was used (calibrated according to the internal calibration plan) which was initially tested for its capability, by using a repeatability and reproducibility study (gage R\&R study). 
Many Statistical Process Control software packages that calculate capability, produce control charts and perform measurement system analysis are available, one of the most common and simple being Minitab software. The analysis in this paper was performed with Minitab ${ }^{\circledR} 17$, one of the most used software for quality improvement.

\subsection{Results and remarks on capability studies}

\subsubsection{Measurement system capability}

When quantifying process capability is important to assure that measurement system is capable. In this study, a R\&R analysis was performed, respectively three inspectors measured three times with a digital caliper (resolution of $0.01 \mathrm{~mm}$ ), the outer diameter of 10 "rubber bushing with mold bonded metal sleeves" (part number 14001), which had been selected to represent the full range of the manufacturing variation.

To assess measurement system capability (total gage $R \& R$ ), the calculated specific metrics were calculated according to AIAG guidelines [2] and are presented in table 1.

- Variance component (VarComp) is used to assess the variation contributed by each source of measurement error relative to the total variation; in a good measurement system, the largest component of variation is part-to-part variation;

- \%Contribution (of VarComp) is based on the variance component estimates; when $\%$ Contribution for part-to-part is high and for gage R\&R as small as possible (less than 1\%;), the measurement system is able to distinguish differences between parts;

- \%Study Variation and/or \%Tolerance; gage R\&R may be calculated whether as a percent of study variation (\%StudyVar) or as a percent of tolerance (\%Tolerance) and must be less than $1 \%$ for a good measurement system. If the analysis is used for process improvement (reducing part-to-part variation), \%StudyVar is a better estimate of measurement system capability and if it is used to evaluate parts relative to specifications, \%Tolerance is a more appropriate metric;

- Number of distinct categories, tells how many separate groups of parts the measurement system is able to distinguish (it must be minimum 5).

In this study, according to AIAG guidelines [2], the measuring system contributes very little to the overall variation, as confirmed by gage $R \& R$ results from table 1 , because:

Table 1. Gage R\&R study $-X b a r / R$ method

\begin{tabular}{|c|c|c|c|c|}
\hline Source & VarComp & $\begin{array}{c}\text { \%Contribution } \\
\text { (of VarComp) }\end{array}$ & $\begin{array}{c}\text { Study Variation } \\
\text { (6xStandDev) }\end{array}$ & $\begin{array}{c}\text { \%Study Variation } \\
\text { (\%StudyVar) }\end{array}$ \\
\hline Total gage R\&R & 0.0000126 & 0.63 & 0.021264 & 7.96 \\
\hline Repeatability & 0.0000126 & 0.63 & 0.021264 & 7.96 \\
\hline Reproducibility & 0.0000000 & 0.00 & 0.0000000 & 0.00 \\
\hline Part-to-Part & 0.0019691 & 99.37 & 0.266247 & 99.68 \\
\hline Total Variation & 0.0019817 & 100.00 & 0.267095 & 100.00 \\
\hline \multicolumn{5}{|l}{ Number of Distinct Categories (ndc) } \\
\hline
\end{tabular}


- The variation due to the measuring system as a \%Contribution is $0.63 \%$, within acceptable range of less than $1 \%$; therefore, $99.37 \%$ of the total variation in the measurements is due to the differences between parts;

- The \%StudyVar results (7.96) indicate that the measurement system accounts for less than $10 \%$ of the overall variation in this study. For this case study, $7.96 \%$ of the variation is estimated to be due to repeatability and $0 \%$ to reproducibility.

- The number of distinct categories is 17 , which indicates the measurement system can distinguish between parts extremely well.

Based on AIAG guidelines [2] this measurement system is acceptable (adequate), meaning that the measurement system is capable to consistently record the same measurement of the outer diameter on this specific part.

\subsubsection{Swaging machine capability}

The study of the swaging machine capability is a preliminary analysis which refers exclusively to the ability of the machine to produce rubber bushing with mold bonded metal sleeves (part number 14001) within required tolerances.

Data of 50 rubber bushings were collected from a continuous, uninterrupted production run of 300 parts on the swaging machine; because it is a calibration with sectors, for each part were used two values, the minimum and maximum to determine an average value of the outer diameter. Design specifications for this characteristic are 25.25 and $25.45 \mathrm{~mm}$.

The capability of the swaging machine has been performed in Minitab (Fig. 2).

Mean $=26,342$

Standard deviation $=0,006468$

Median $=26,34$

Skewness $=0,2003$

Kurtosis $=-0,7105$

$\mathrm{Cm}=5.15$

Cmk=4.74

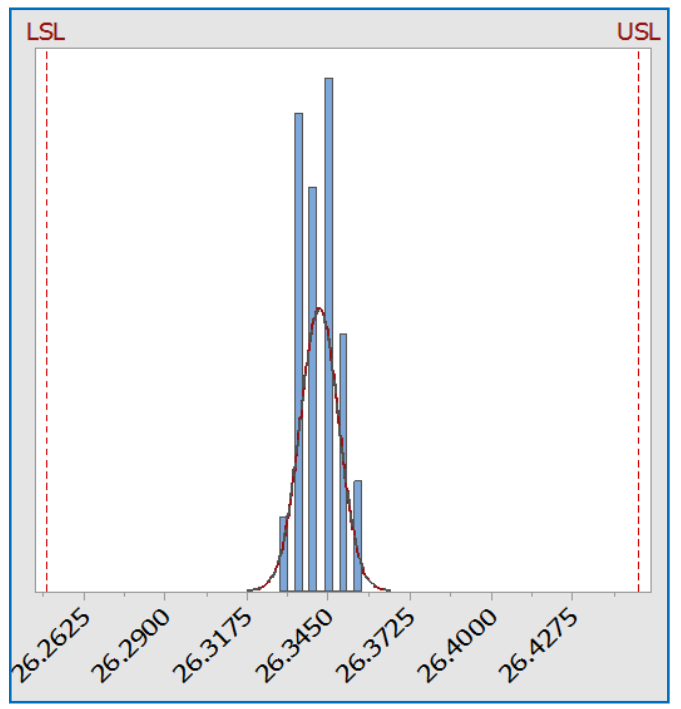

Fig. 2. Swaging machine capability

The capability of swaging machine is evaluated using the indices of capability $\mathrm{Cm}$ and $\mathrm{Cmk}$, where $\mathrm{Cm}$ indicates variance and $\mathrm{Cmk}$ position. A normal requirement is that $\mathrm{Cmk}$ should be at least 1.67 .

The capability of the swaging machine is very high $(\mathrm{Cmk}=4.74)$; this means that it is a good machine with a small spread in relation to the tolerance with.

The slight difference between $\mathrm{Cm}(\mathrm{Cmk}=5.15)$ and $\mathrm{Cmk}$ indicate that the results are not perfectly centered but are slightly displaced to the lower specification limit.

High values of these indices are synonymous with good quality and high yield of the swaging machine under investigation. 


\subsubsection{Preliminary process capability}

In the automotive industry, the Production Part Approval Process (PPAP) states "An acceptable level of preliminary process capability must be determined prior to submission for all characteristics designated by the customer or supplier as safety, key, critical, or significant, that can be evaluated using variables (measured) data."

Because the paper presents a process for a new product, a preliminary process capability study (short term study) was necessary to obtain early information on the performance of the swaging process relative to requirements. To determine the preliminary capability, 50 parts were randomly collected from the same parts produced for preparation of PPAP file.

Before estimating preliminary proces capability, two assumptions have been verified: the quality characteristic has a normal distribution and observations are random and independent of each other.

Figure 3 shows normal probability plot for the case study data, performed with Minitab: mean is 26.34, standard deviation is 0.006468 , Ryan-Joiner test statistic value is 0.997 and P-value is greater 0.100 so more than the significance level $(\alpha=0.05)$ wich implies that the data is distributed normally. Thus, it was concluded that the data appears to be normal.

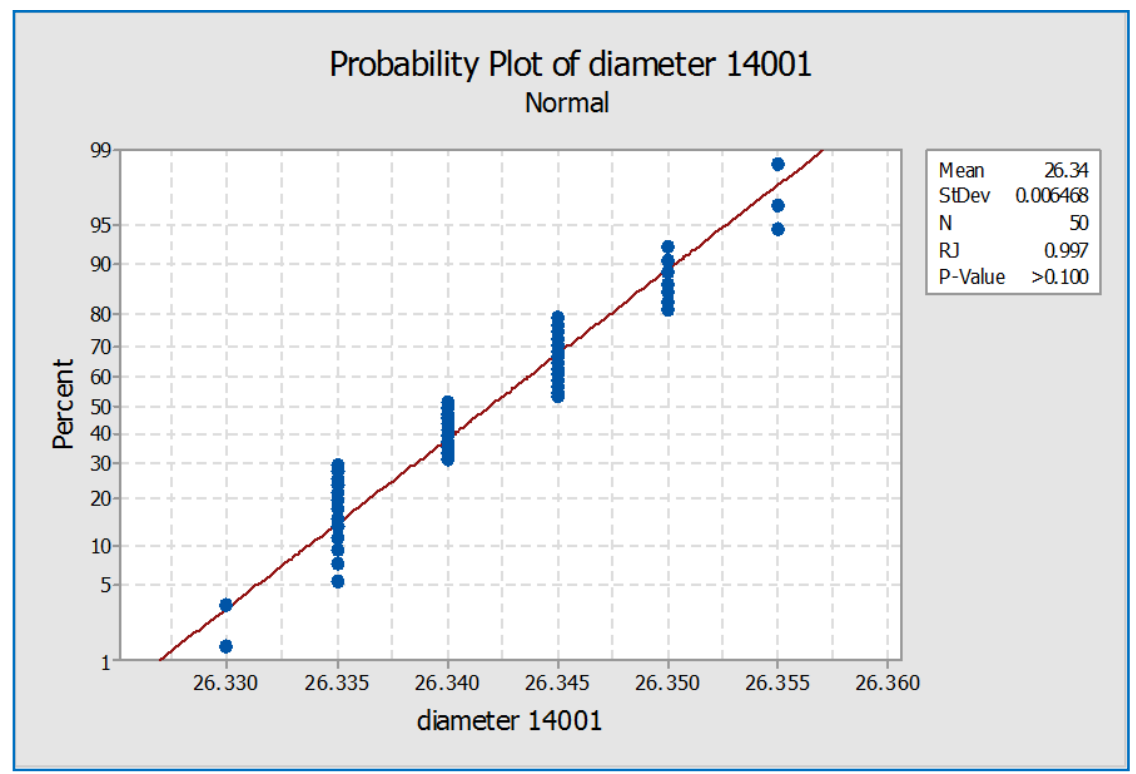

Fig. 3. Test for checking the normality assumption

Figure 4 presents the runs test, a nonparametric test for checking the assumption of randomness using Minitab software.

We see that the P-values for clustering, mixtures, trends and oscillation are greater than alpha value of 0.05 and the actual numbers of runs are close to the expected number of runs, so it was concluded that the data is independent and random.

After validating these critical assumptions, preliminary capability indices Pp and Ppk were calculated and were obtained the results shown in figure 5. It is noted that the swaging process has a preliminary capability that meets the requirements: Ppk $=4.11>1.67$ and PPM $=0$.

In addition, values of $\mathrm{Pp} \mathrm{(4.37)}$ and Ppk (4.11) very close indicate that the process is well centered. 


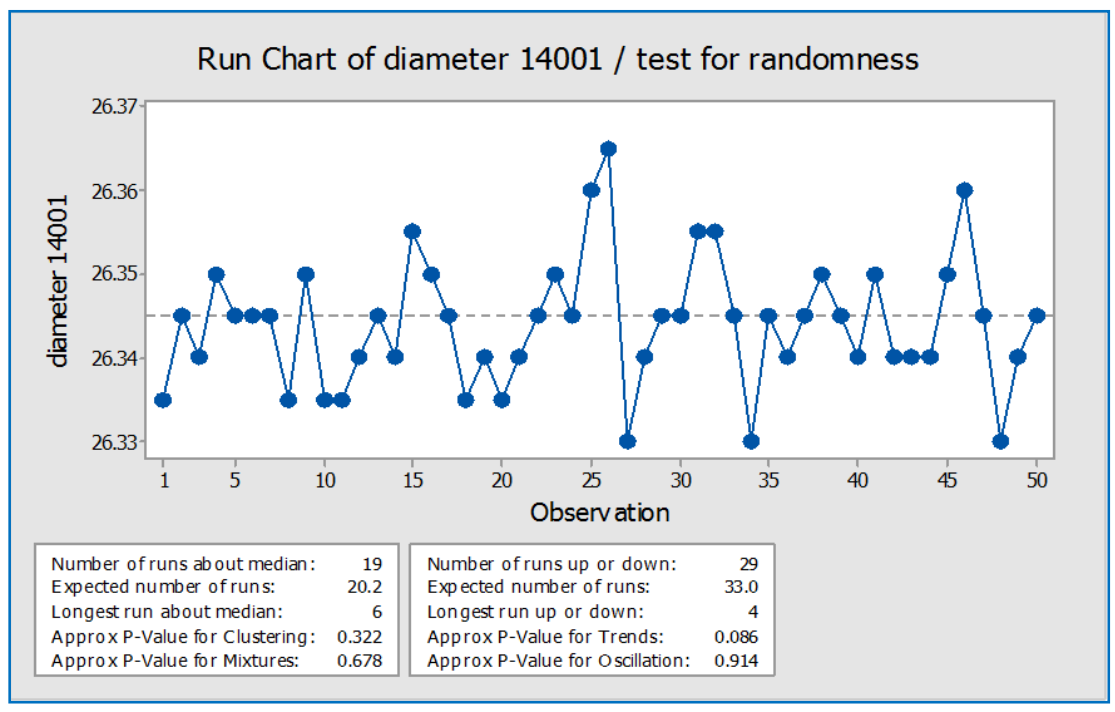

Fig. 4. Test for randomness

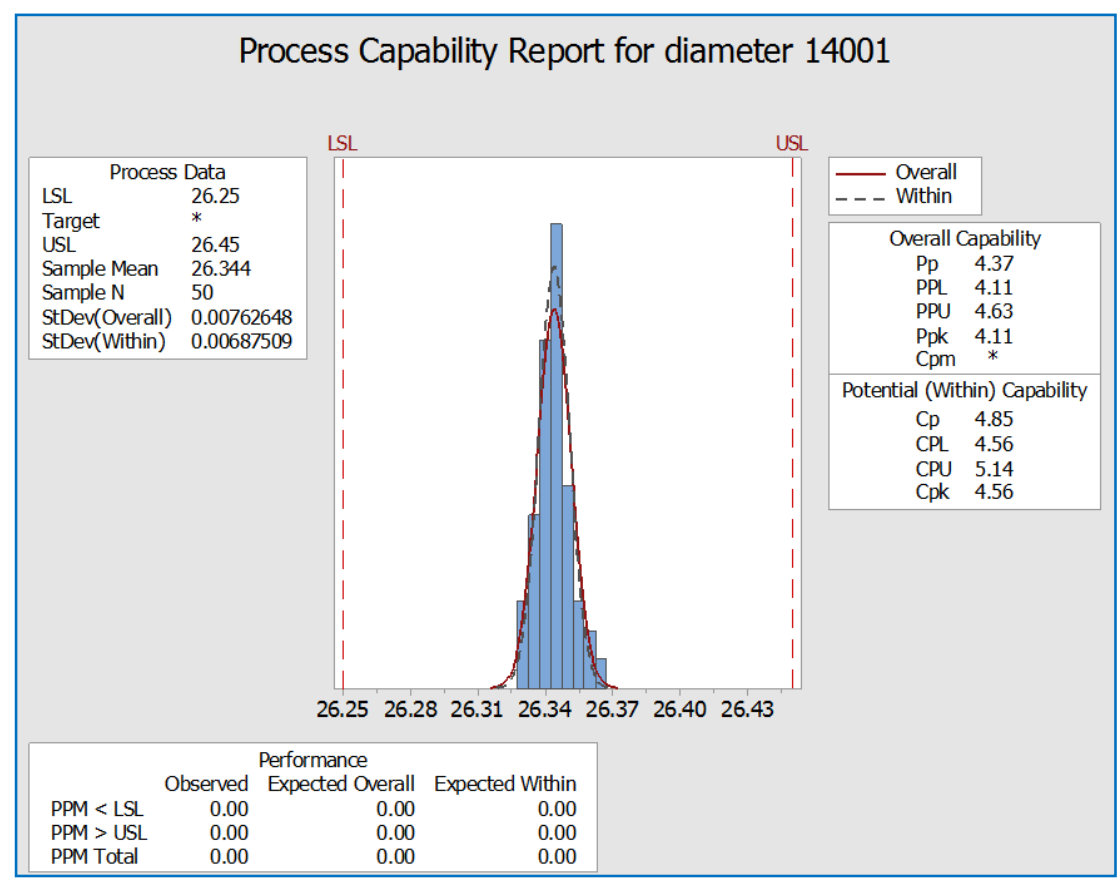

Fig. 5. Preliminary process capability

\subsubsection{Process capability and performance}

Capability and performance of the calibration process were analyzed during the first production serie for the rubber bushing with mold bonded metal sleeves (part number 14001), when there were vulcanized and then calibrated a number of 20000 parts.

For this analysis, 24 subgroups have been collected each hour, during three different work shifts to include as many potential sources of variation; the subgroup size is 5 parts. 
There are two critical assumptions to consider when performing process capability analyses [13]: the output data must follow a normal distribution and the process must be in statistical control (stable).

To ensure that the data is normal distributed, it was conducted again a normality test whose result was positive, the value of $\mathrm{p}$ being greater than 0.10 .

To assess whether the data is statistically stable (in statistical control), with no special causes of variation present, a control chart by variables was completed (Fig. 6).

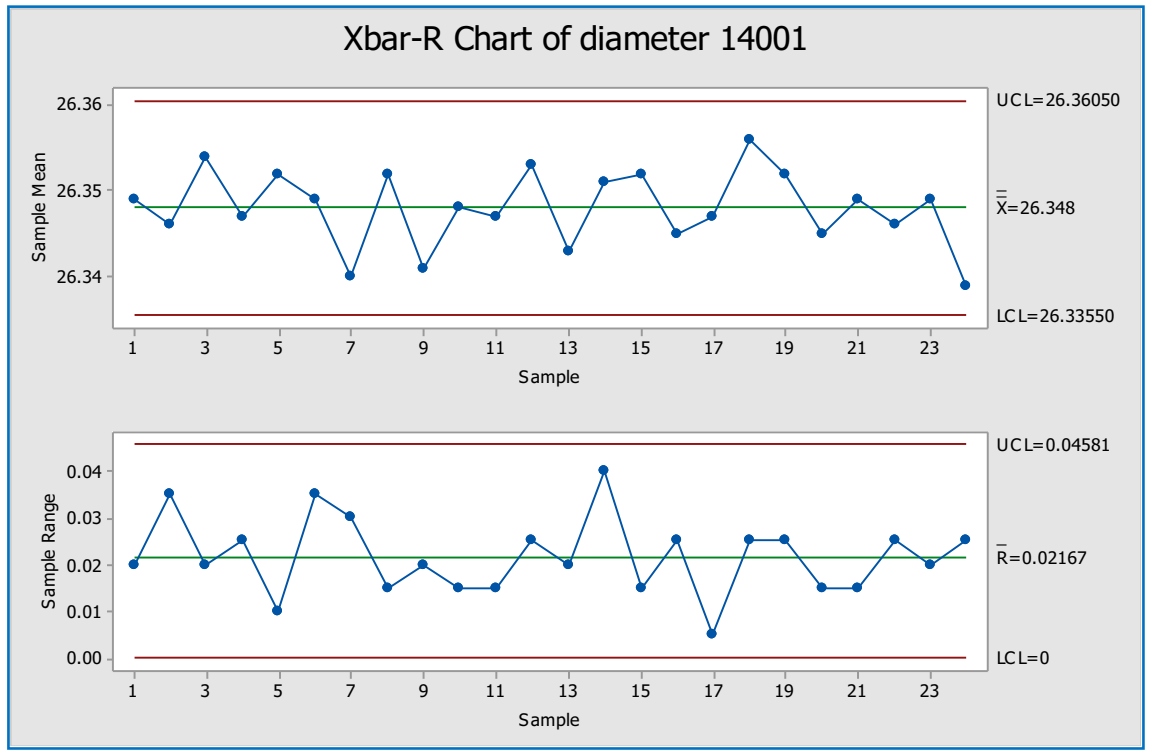

Fig. 6. Control chart by variables for case study data

From figure 6 it can be observed that all plotted subgroup mean and range values are within the control limits on both as XBar chart as well as R chart and no indication of trend, shift, run and clustering has been depicted.

So, the conclusion is that the swaging process is under statistical control and operating under the influence of only chance causes of variation, i.e. the process is stable over time both as adjustment and precision.

After validating the critical assumptions on the swaging process (normally distributed and under statistical control) overall capability analysis was performed, and the results are present in figure 7. Based on the results, the following observations can be made:

- The process is capable on short term, with capability indices: $\mathrm{Cp}=3.52 / \mathrm{Cpk}=3.45>1.33$

- The process is capable on long term, with performance indices: $\mathrm{Pp}=3.50 / \mathrm{Ppk}=3.43>1.67$, so the process is performant;

- The process meets inclusive the requirement for six sigma, $\mathrm{Cpk} \geq 2.00$;

- $\mathrm{Cp}$ and $\mathrm{Cpk}$ values, respectively $\mathrm{Pp}$ and Ppk, very close indicate that the process is well centered;

- Cpk and Ppk values almost identical, show that process variation is due only to the random causes and no systematic variation occur, so the process is stable;

- No single index should be used to describe a process. For a better understanding of the process and to carry out correct decisions, it is strongly recomended that all for indices $(\mathrm{Cp}$, Cpk and Pp, Ppk) be calculated and compared on the same data set;

- Parts per Million (PPM) is zero. This index indicates the ratio between the number of pieces exceeding the specifcation limits and a million produced units and his value represents the loss of quality due to special causes. 
In conclusion, the results showed that we are dealing with a normaly distributed and stable process that comply with customer requirements both short-term and long-term.

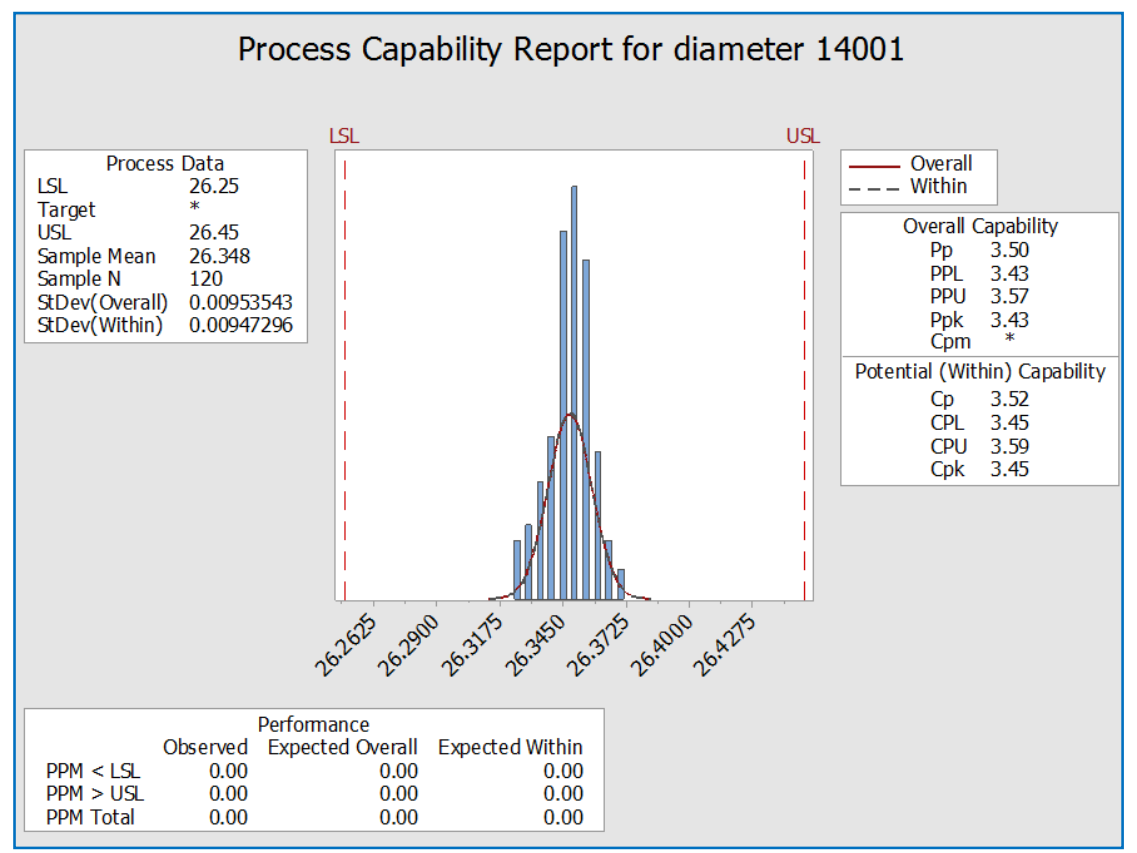

Fig. 7. Process capability and performance

If capability analysis is performed, $\mathrm{Pp}$ and Ppk indices will show approximately what happened in the past, but cannot be used to predict capability in the future. It will provide only a snapshot of the process at best. If, however, a system is stable, capability analysis through $\mathrm{Cp}$ and $\mathrm{Cpk}$ indices shows not only the ability of the system in the past, but also, if the system remains stable, predicts the future performance of the system.

\section{Conclusions}

The aim of this study was to highlight what powerful tools are capability studies for monitoring and ensuring the quality of products to satisfy the customer requirements, but also the importance of correct understanding, use and interpretation of capability indices.

This knowledge is necessary for controlling the process efficiently and effectively so that the products realized by the process fulfil the quality requirement.

No single index should be used to describe a process. For a better understanding of the process and to carry out correct decisions, it is strongly recomended that more indices be calculated and compared on the same data set.

Before quantifying the capability indices, validation of certain critical assumptions which allow the use of such metrics to have a meaningful interpretation, but that are frequently overlooked, was realized with the help of some quality statistical tools like normal probability plot, run chart and control charts by using the Minitab software.

The case study was conducted in a company activating in the automotive industry from Sibiu area, expert in automotive anti-vibration systems, to show the importance of capability studies in manufacturing process control. The automotive industry belongs to one of the largest industries and their manufacturing systems are placed before an increasingly fierce competition, within which the analysis of processes by the use of capability and performance 
indices came to play a key role since they assist to decide whether a manufacturing process is suitable to satisfy the customer quality requirements.

\section{References}

1. D. C. Montgomery, Introduction to Statistical quality control, Sixth edition (John Wiley \& Sons, Inc., New York, 2009)

2. Automotive Industry Action Group, AIAG, Measurement Systems Analysis, Reference Manual, Fourth edition (Detroit-Michigan, USA, June 2010)

3. M. Mottonen, P. Belt, J. Harkonen, H. Haapasalo, P. Kess, The O. Ind. \& Man. Eng. J. 1, 29 (2008)

4. ISO 22514-1 Statistical methods in process management. Capability and performance - Part 1: General principles and concepts (2014)

5. https://elsmar.com/pdf_files/CPK.pdf, Accessed: 01.03.2017

6. Automotive Industry Action Group, AIAG, Statistical Process Control, Reference manual, Second edition (Detroit-Michigan, USA, 2005)

7. ISO/TS 16949 Quality management systems. Particular requirements for the application of ISO 9001:2000 for automotive production and relevant service part organizations (2009)

8. $\quad$ S. P. Wanare, M. V. Gudadhe, IJIET, 2 (2), 198 (2013)

9. https://wenku.baidu.com/view/12db0ffd04a1b0717fd5dd3e.html, Accessed: 01.03.2017

10. ISO 22514-7 Statistical methods in process management. Capability and performance - Part 7: Capability of measurement processes (2016)

11. http://www.world-classquality.com/images/article/20080806154501_Initial\%20process\%20capability\%20QW\%20Feb0 7.pdf, Accessed: 05.03.2017

12. ISO 22514-4 Statistical methods in process management. Capability and performance - Part 4: Process capability estimates and performance measures (2016)

13. https://www.minitab.com/uploadedFiles/Content/News/Published_Articles/capability_analysis_1 .pdf, Accessed: 05.03.2017

14. http://www.symphonytech.com/articles/processcapability.htm, Accessed: 05.03.2017 